\title{
GaN changes the game in power conversion
}

TECHNOLOGY ADVANCES seeks materials developments on the threshold of commercialization.

Send suggestions to MRS Bulletin at Bulletin@mrs.org

\section{The pitch}

The form of electrical energy that is required depends strongly on the function to be performed. For example, a laptop computer typically needs $18 \mathrm{~V}$ direct current $(\mathrm{dc})$. The conversion from the $110 \mathrm{~V}$ alternating current (ac) outlet to the $18 \mathrm{~V} \mathrm{dc}$ is performed by a voltage converter. The dc power from a photovoltaic panel needs to be converted to ac to feed into the grid by a circuit called an inverter. Such a dc-to-ac power conversion also occurs in hybrid cars where dc energy from the battery is converted to ac energy to drive the electric motors. Consequently power conversion is ubiquitous with an annual market value of over $\$ 7$ billion growing at a compound annual growth rate of over $12.6 \%$.

The devices being used for power conversion are Si-based diodes and transistors, predominantly metal oxide semiconductor field-effect transistors and insulated gate bipolar transistors. While Si has performed well in the past, it has reached its material limits for power conversion. Thus increases in efficiency are becoming more difficult to achieve. Inefficient electric power conversion results in hundreds of terawatts of lost energy across the electrical grid in the United States, equivalent to 318 coal-fired power plants. The cost to the U.S. economy is $\$ 40$ billion annually, which represents over $10 \%$ of all the power generated in the United States today. The losses in electrical power conversion are larger than all the electricity produced from the alternative energy sources of power combined by over an order of magnitude. To eliminate these losses requires moving to a new materials system to produce an ideal switch, that is, the ability to hold large voltages when off and dissipate negligible loss when passing current in the on state.

According to the company Transphorm, the optimum and most economical materials to perform this function are based on gallium nitride. Transphorm's GaN-based power solutions increase efficiency, reduce system size, and simplify overall product design and can eliminate up to $90 \%$ of all electric conversion losses from heating, ventilation, and air-conditioning systems (HVAC) and solar panels.

\section{The technology}

The power-conversion devices that are

\section{Surface analysis upgrade for your AFM}

Force curve at every image pixel

Surface property maps from arbitrary force model

Use standard cantilevers, scan at standard speed

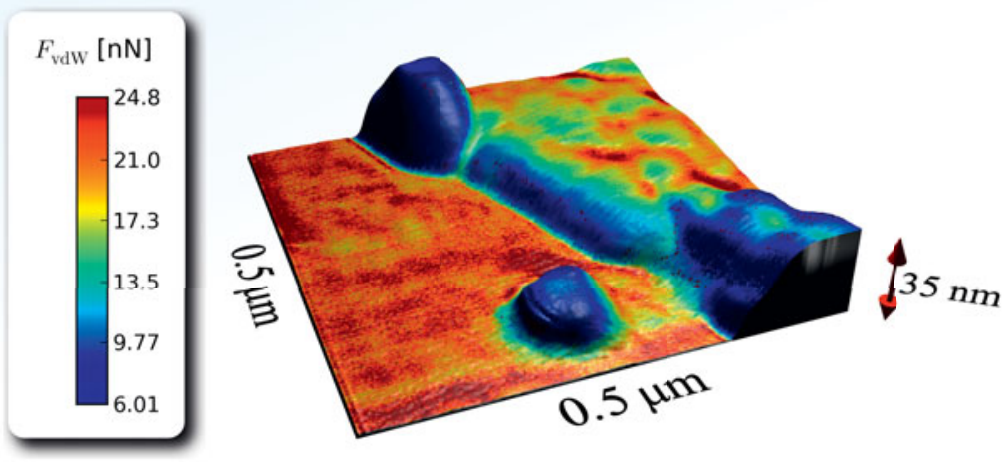

The van der Waals attractive force mapped on the surface topography. Step edge of a thin film of butylacrylate spin coated on silicon oxide. 


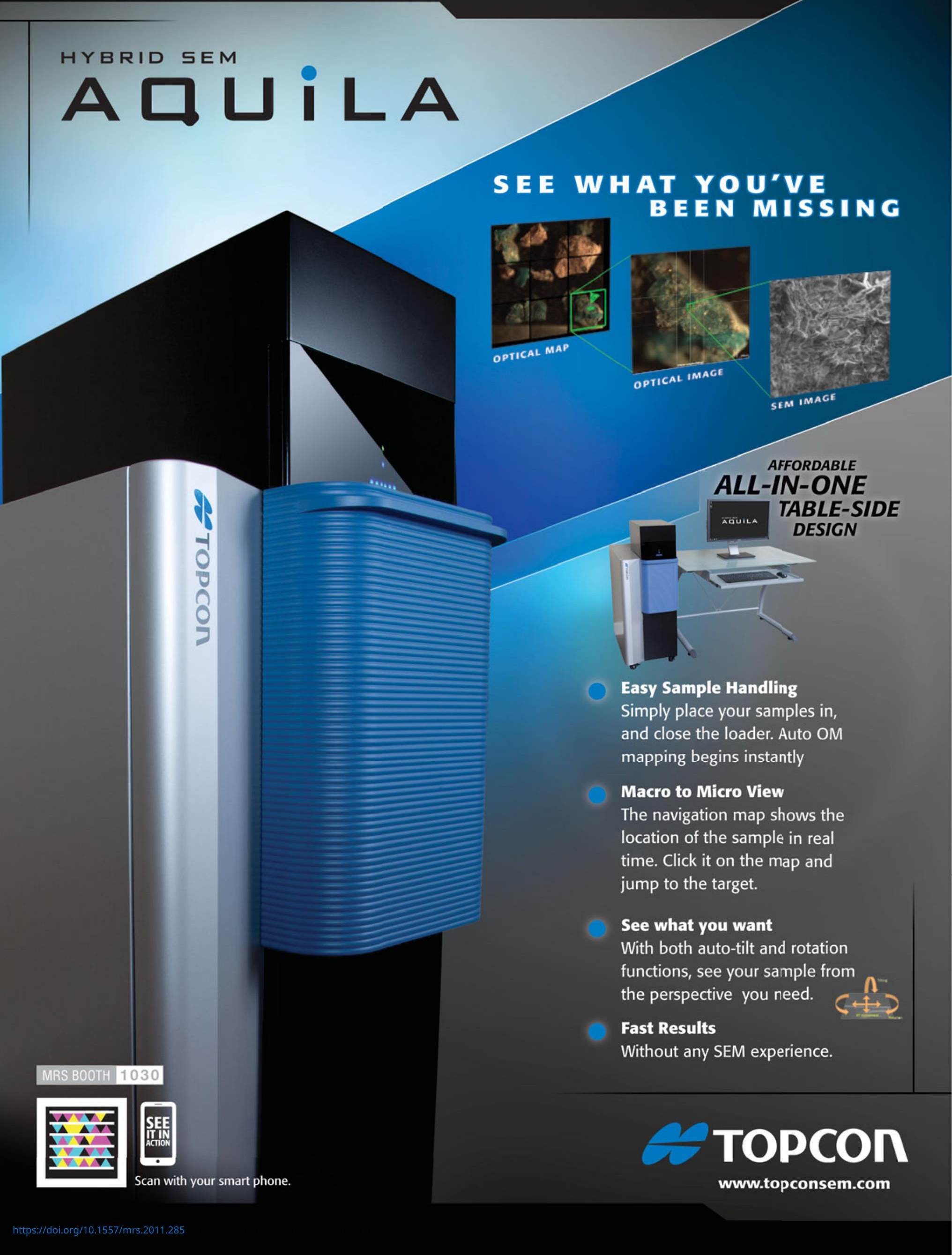




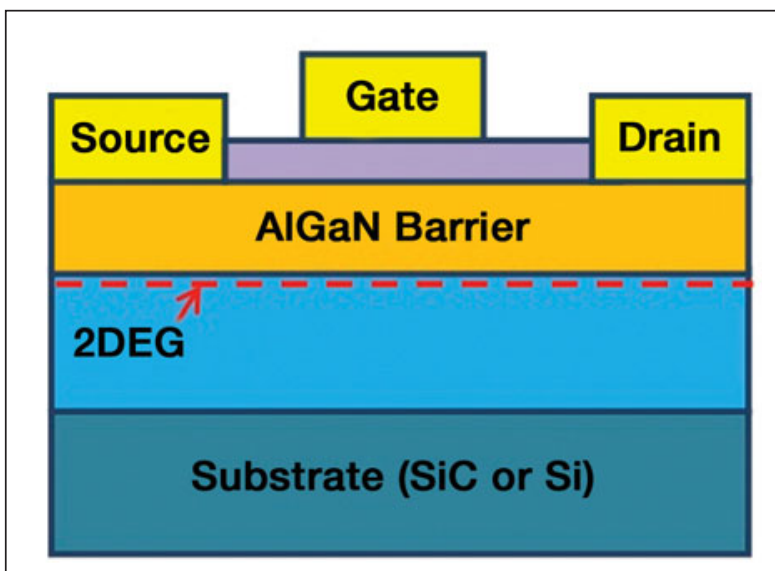

The AIGaN/GaN heterostructure used to fabricate Transphorm's diodes and transistors. The highelectron-mobility channel is also shown. possessing high electron mobility formed at the $\mathrm{AlGaN} / \mathrm{GaN}$ interface to neutralize the positive charge that exists at the interface due to the polarization difference between the AlGaN and the GaN. This polarization difference (a materials property) increases almost linearly with $\mathrm{Al}$ composition enabling a simple tailoring of the electron density by material composition without the need for doping. This results in

integrated into the final module solutions are based on the aluminum gallium nitride $(\mathrm{AlGaN}) /$ gallium nitride $(\mathrm{GaN})$ heterostructure fabricated into both diodes and transistors. The transistor is a high-electron-mobility transistor. The cross section of the material structure and the device design of a typical device is shown in the figure. The current is carried by a two-dimensional electron gas extremely high electron mobility, in excess of $2000 \mathrm{~cm}^{2} \mathrm{~V}^{-1} \mathrm{~s}^{-1}$ with charge densities typically around $1 \times 10^{13} \mathrm{~cm}^{-2}$. The resulting low resistance of the channel coupled by the high breakdown voltage enabled by the high breakdown field strength $\left(>3 \times 10^{6} \mathrm{~cm}^{-1} ; 10\right.$ times that of silicon because of the larger bond strength and bandgap of $\mathrm{GaN}$ ) results in exceptionally high efficiencies of over
$99.2 \%$ in converting $200 \mathrm{~V}$ dc to $400 \mathrm{~V}$ $\mathrm{dc}$ and $98.5 \%$ in a photovoltaic inverter both in the range of a $\mathrm{kW}$ of power at a high frequency of $100 \mathrm{kHz}$. These results demonstrate that the promise of highefficiency GaN-based power conversion is now becoming a reality and the market penetration will continue to increase as the technology matures and the advantages of low-loss and small form factor drive new designs.

\section{Opportunities \\ Transphorm is currently working with and continues to seek to work with companies as customer-partners to help develop new solutions together by combining Transphorm's expertise with that of the customer. The company also works with universities that have an established expertise in power conversion so that the capabilities of the technology can be studied in innovative new architectures and applications including advanced packaging. \\ Source: Carl Blake, VP Marketing, Transphorm Inc., 115 Castilian Drive, Goleta, CA 93117, USA; tel. 805-456-1300 ext.116; fax 805-968-1985; email cblake@transphormusa.com; and www.transphormusa.com.}

\section{Seeing more clearly at the nanoscale}

\section{The pitch}

Recent advancements in a wide range of instrumentation including high-resolution electron and optical microscopes, chemical analyzers, and spectroscopes allow researchers to see and manipulate matter with unprecedented precision and accuracy. However, despite large investments by universities, government facilities, and industry in these instruments and capabilities, sample preparation and poor sample quality continue to impede characterization accuracy, reproducibility, and throughput. Poor sample preparation creates artifacts such as aggregation or sample damage that introduce uncertainty in data and analysis and that often require multiple samples for a single quality data point or image.
Dune Sciences' patent-pending SMART grids $^{\mathrm{TM}}$ functionalized characterization substrates address this problem by imparting greater control over specimen dispersion, coverage, uniformity, and repeatability. In addition, SMART grids facilitate correlated analysis using multiple analytical instruments on the same sample to streamline the characterization process. SMART grids and related characterization products reduce the time and money required for sample preparation and analysis by $50 \%$ or more in many cases and result in increased confidence in reporting results due to improved data quality.

The total market for analytical instruments for nanoscale characterization including electron, ion, atomic force and optical microscopes, surface/ chemical analyzers, and other equipment exceeded \$3 billion in 2009. Dune Sciences' products and services leverage the value of these instruments by maximizing their utility to meet a growing list of application needs in both materials science and life science. In materials science SMART grids standardize sample preparation and provide superior data quality for a wide range of materials for accurate determination of their physical properties (e.g., size and shape), for process optimization and materials integration, manufacturing quality control, failure analysis, and environmental monitoring of nanomaterials to determine their fate and transport. In the life sciences they enhance sample specificity and reproducibility for higher throughput analysis in structural biology, toxicology, pharmaceutical research, and quality control and diagnostic screening (i.e., viral/bacterial). 